\title{
The CPT group of the spin-3/2 field
}

\author{
B. Carballo Pérez*and M. Socolovsky ${ }^{\dagger}$ \\ Instituto de Ciencias Nucleares, Universidad Nacional Autónoma de México, \\ Circuito exterior, Ciudad Universitaria, 04510, México D.F., México
}

\begin{abstract}
We find out that both the matrix and the operator CPT groups for the spin-3/2 field (with or without mass) are respectively isomorphic to $D_{4} \rtimes \mathbb{Z}_{2}$ and $Q \times \mathbb{Z}_{2}$. These groups are exactly the same groups as for the Dirac field, though there is no a priori reason why they should coincide.
\end{abstract}

Keywords: discrete symmetries; spin 3/2-field; finite groups

*brendacp@nucleares.unam.mx

†socolovs@nucleares.unam.mx 


\section{Introduction}

The CPT group of the Dirac field in Minkowski space-time was obtained by Socolovsky in 2004 [1]. It were found two sets of consistent solutions for the matrices of charge conjugation $(C)$, parity $(P)$, and time reversal $(T)$, which give the transformation of fields $\hat{\psi}_{C}(x)=C \hat{\bar{\psi}}^{T}(x), \hat{\psi}_{\Pi}\left(x_{\Pi}\right)=P \hat{\psi}(x)$ and $\hat{\psi}_{\tau}\left(x_{\tau}\right)=$ $T \hat{\psi}(x)^{*}$, where $x_{\Pi}=(t,-\mathbf{x})$ and $x_{\tau}=(-t, \mathbf{x})$. These sets are given by:
a) $C_{D}= \pm \gamma^{2} \gamma^{0}, P_{D}= \pm i \gamma^{0}, T_{D}= \pm i \gamma^{3} \gamma^{1}$
b) $C_{D}= \pm i \gamma^{2} \gamma^{0}, P_{D}= \pm i \gamma^{0}, T_{D}= \pm \gamma^{3} \gamma^{1}$.

Each of these sets generates a non abelian group of sixteen elements, respectively, $G_{\theta}^{(1)} \cong D_{4} \times \mathbb{Z}_{2}$ and $G_{\theta}^{(2)} \cong 16 E$, where $D_{4}$ is the group of symmetries of the square and $16 E$ is a non trivial extension of $D_{4}$ by $\mathbb{Z}_{2}$, isomorphic to a semidirect product of these groups.

On the other hand, the quantum operators $\hat{C}, \hat{P}$ and $\hat{T}$, acting on the Hilbert space, generate a unique group $G_{\hat{\theta}} \cong Q \times \mathbb{Z}_{2}$, where $Q$ is the quaternion group.

With this in mind, we decided to find the $C P T$ group of the spin-3/2 field (Rarita-Schwinger field), for both massive and massless cases. This field could be useful for the description of compound objects (neglecting its structure in a first approximation), like the baryon decuplet components for spin-3/2 $[2]$, or for elementary fields such as the gravitino.

In order to describe 3/2-spin particles, the set of equations

$$
\begin{aligned}
\left(i \gamma^{\alpha} \partial_{\alpha}-m\right) \hat{\psi}^{\mu}(x) & =0, \\
\gamma^{\mu} \hat{\psi}_{\mu}(x) & =0,
\end{aligned}
$$

where $\partial_{\alpha}=\frac{\partial}{\partial x_{\alpha}}$, is required. These equations are known as the Rarita-Schwinger equation [3]. The first of these equation is a Dirac equation for each vector component of the vector spinor $\hat{\psi}^{\mu}$ and the second one is known as the subsidiary condition. Precisely due to the more complexity of these equations with respect to the Dirac field equation, there is not a priori any apparent reason why the CPT group for the Rarita-Schwinger field should coincide with those for the Dirac field.

\section{Parity}

If we want to study the $P$ invariance of the Rarita-Schwinger equation, we need to repeat the analysis done for the Dirac equation in [1] and also consider the $P$ invariance of the subsidiary equation. 
Multiplying this equation from the left by $P$, changing $\mathbf{x} \rightarrow-\mathbf{x}$ and inserting the unity, one obtains:

$$
\begin{array}{r}
P \gamma^{0} P^{-1} P \hat{\psi}_{0}(t,-\mathbf{x})+P \gamma^{1} P^{-1} P \hat{\psi}_{1}(t,-\mathbf{x})+P \gamma^{2} P^{-1} P \hat{\psi}_{2}(t,-\mathbf{x}) \\
+P \gamma^{3} P^{-1} P \hat{\psi}_{3}(t,-\mathbf{x})=0
\end{array}
$$

but we need to take into account that the vector spinor changes by parity in the following way:

$$
\hat{\psi}_{\mu}(t, \mathbf{x})=\left(\begin{array}{c}
\hat{\psi}_{0}(t, \mathbf{x}) \\
\hat{\psi}_{1}(t, \mathbf{x}) \\
\hat{\psi}_{2}(t, \mathbf{x}) \\
\psi_{3}(t, \mathbf{x})
\end{array}\right) \longrightarrow \hat{\psi}_{\mu \pi}(t, \mathbf{x})=\left(\begin{array}{c}
P \hat{\psi}_{0}(t,-\mathbf{x}) \\
-P \hat{\psi}_{1}(t,-\mathbf{x}) \\
-P \hat{\psi}_{2}(t,-\mathbf{x}) \\
-P \psi_{3}(t,-\mathbf{x})
\end{array}\right)
$$

where $\hat{\psi}_{\mu \pi}(t, \mathbf{x})$ can be written as $\hat{\psi}_{\mu \pi}(t, \mathbf{x})=\mathcal{P} P \hat{\psi}_{\mu}(t,-\mathbf{x})$, with $\mathcal{P} \in O(1,3)$ given by:

$$
\mathcal{P}=\left(\begin{array}{cccc}
1 & 0 & 0 & 0 \\
0 & -1 & 0 & 0 \\
0 & 0 & -1 & 0 \\
0 & 0 & 0 & -1
\end{array}\right)
$$

and $P \in D^{16}$, where $D^{16}$ is the Dirac algebra.

Substituting the components of $\hat{\psi}_{\mu \pi}(t, \mathbf{x})$ in (3) one obtains:

$$
P \gamma^{0} P^{-1} \hat{\psi}_{0 \pi}(t, \mathbf{x})-P \gamma^{k} P^{-1} \hat{\psi}_{k \pi}(t, \mathbf{x})=0 .
$$

This implies the constraints on $P$ :

$$
P \gamma^{0} P^{-1}=\gamma^{0}, \quad P \gamma^{k} P^{-1}=-\gamma^{k}
$$

or

$$
P \gamma^{0} P^{-1}=-\gamma^{0}, \quad P \gamma^{k} P^{-1}=\gamma^{k}
$$

The relations (7) are the same as those obtained from the Dirac equation, whose already known solution is $P_{D}= \pm i \gamma^{0}$, while from the relations (8) is obtained $P=P^{\prime}=z \gamma^{3} \gamma^{2} \gamma^{1}$, with $z \in \mathbb{C}^{*}=\mathbb{C} \backslash\{0\}$.

Following the same analysis as in [1], there are two possibilities for each $P$ : 

a) $P^{2}=+1 \Rightarrow z= \pm 1 \Rightarrow P^{\prime}= \pm \gamma^{3} \gamma^{2} \gamma^{1}$;
b) $P^{2}=-1 \Rightarrow z= \pm i \Rightarrow P^{\prime}= \pm i \gamma^{3} \gamma^{2} \gamma^{1}$.

In the first case: $P^{\prime \dagger}=P^{\prime}=P^{\prime-1}=-P^{\prime T}=-P^{\prime *}$ and in the second one: $P^{\prime \dagger}=-P^{\prime}=P^{\prime-1}=P^{\prime T}=-P^{\prime *}$.

\section{Charge conjugation}

To study the $C$ invariance of the subsidiary equation, we must take the complex conjugate of this equation, multiply from the left by $C \gamma_{0}$ and insert the unit matrix. This is:

$$
\left(C \gamma^{0}\right) \gamma^{\mu *}\left(C \gamma^{0}\right)^{-1} \hat{\psi}_{\mu C}(x)=0
$$

where $\hat{\psi}_{\mu C}(x)=C \gamma^{0} \hat{\psi}_{\mu}^{*}(x)$.

In this case, the constraints on $C$ are:

$$
\left(C \gamma^{0}\right) \gamma^{\mu *}\left(C \gamma^{0}\right)^{-1}=\gamma^{\mu}
$$

or

$$
\left(C \gamma^{0}\right) \gamma^{\mu *}\left(C \gamma^{0}\right)^{-1}=-\gamma^{\mu}
$$

Taking into account that $\gamma^{0} \gamma^{\mu *} \gamma^{0}=\gamma^{\mu T}$, one can find the solutions for the $C$ matrices. The equation with the negative sign is the same as in the Dirac case and leads to $C=C_{D}=\eta \gamma^{2} \gamma^{0}$; on the other hand, from equation (10) we arrive to:

$$
C \gamma^{\mu T} C^{-1}=\gamma^{\mu}
$$

which leads to the solution $C=C^{\prime}=\eta \gamma^{3} \gamma^{1}$.

For $C^{\prime}$, a second application of the charge conjugation transformation is given by:

$$
\begin{aligned}
\left(\hat{\psi}_{C}\right)_{C} & =\hat{\psi}_{C^{2}}=C^{\prime} \hat{\bar{\psi}}_{C}^{T}=C^{\prime}\left(\hat{\psi}_{C}^{\dagger} \gamma^{0}\right)^{T}=C^{\prime} \gamma^{0} \hat{\psi}_{C}^{*}=C^{\prime} \gamma^{0} C^{\prime *} \gamma^{0} \hat{\psi}=-C^{\prime} C^{\prime *} \hat{\psi} \\
& =-|\eta|^{2} \gamma^{3} \gamma^{1} \gamma^{3 *} \gamma^{1 *} \hat{\psi}=|\eta|^{2}\left(\gamma^{3}\right)^{2}\left(\gamma^{1}\right)^{2} \hat{\psi}=|\eta|^{2} \hat{\psi}
\end{aligned}
$$

Since the effect on $\hat{\psi}$ can be, at most, a multiplication by a phase, then $\eta \in$ $U(1)$ and $C^{\prime}$ is unitary. This is: 


$$
C^{\prime} C^{\prime \dagger}=\eta \gamma^{3} \gamma^{1} \bar{\eta}\left(\gamma^{3} \gamma^{1}\right)^{\dagger}=|\eta|^{2} \gamma^{3} \gamma^{1} \gamma^{1} \gamma^{3}=|\eta|^{2} \gamma^{3}\left(\gamma^{1}\right)^{2} \gamma^{3}=|\eta|^{2} 1=1 .
$$

Hence, for $C=C^{\prime}$ we also find that $\hat{\psi}_{C^{2}}=\hat{\psi}$.

As in [1], due to a symmetry consideration between $\hat{\psi}_{C}=C^{\prime} \hat{\bar{\psi}}^{T}$ and $\hat{\bar{\psi}}_{C}=$ $-\bar{\eta}^{2} \hat{\psi}^{T} C^{\prime}$, it follows that $-\bar{\eta}^{2}= \pm 1$ and taking into account that $|\eta|^{4}=1$, one obtains $\eta^{2}= \pm 1$, which implies that $\eta= \pm 1, \pm i$.

Then, for the spin-3/2 field there are two possibilities for each $C$ :
a) $C^{\prime}= \pm \gamma^{3} \gamma^{1}$
$C_{D}= \pm \gamma^{2} \gamma^{0}$
b) $C^{\prime}= \pm i \gamma^{3} \gamma^{1}, \quad C_{D}= \pm i \gamma^{2} \gamma^{0}$.

\section{Time reversal}

We start again from the subsidiary equation, change $t \rightarrow-t$ and take the complex conjugate:

$$
\begin{array}{r}
T \gamma^{0 *} T^{-1} T \hat{\psi}_{0}(-t, \mathbf{x})^{*}+T \gamma^{1 *} T^{-1} T \hat{\psi}_{1}(-t, \mathbf{x})^{*}+T \gamma^{2 *} T^{-1} T \hat{\psi}_{2}(-t, \mathbf{x})^{*} \\
+T \gamma^{3 *} T^{-1} T \hat{\psi}_{3}(-t, \mathbf{x})^{*}=0
\end{array}
$$

but we need to take into account that the vector spinor changes by time inversion in the following way:

$$
\hat{\psi}_{\mu}(t, \mathbf{x})=\left(\begin{array}{c}
\hat{\psi}_{0}(t, \mathbf{x}) \\
\hat{\psi}_{1}(t, \mathbf{x}) \\
\hat{\psi}_{2}(t, \mathbf{x}) \\
\psi_{3}(t, \mathbf{x})
\end{array}\right) \longrightarrow \hat{\psi}_{\mu \tau}(t, \mathbf{x})=\left(\begin{array}{c}
-T \hat{\psi}_{0}(-t, \mathbf{x})^{*} \\
T \hat{\psi}_{1}(-t, \mathbf{x})^{*} \\
T \hat{\psi}_{2}(-t, \mathbf{x})^{*} \\
T \psi_{3}(-t, \mathbf{x})^{*}
\end{array}\right)
$$

where $\hat{\psi}_{\mu \tau}(t, \mathbf{x})$ can be written as $\hat{\psi}_{\mu \tau}(t, \mathbf{x})=\mathcal{T} T \hat{\psi}_{\mu}(-t, \mathbf{x})^{*}$, with $\mathcal{T} \in O(1,3)$ given by:

$$
\mathcal{T}=\left(\begin{array}{cccc}
-1 & 0 & 0 & 0 \\
0 & 1 & 0 & 0 \\
0 & 0 & 1 & 0 \\
0 & 0 & 0 & 1
\end{array}\right)
$$

and $T \in D^{16}$. 
Substituting the components of $\hat{\psi}_{\mu \tau}(t, \mathbf{x})$ in (15) one obtains:

$$
-T \gamma^{0 *} T^{-1} \hat{\psi}_{0 \tau}(t, \mathbf{x})+T \gamma^{k *} T^{-1} \hat{\psi}_{k \tau}(t, \mathbf{x})=0,
$$

from which we can deduce the constraints on $T$ :

$$
T \gamma^{0} T^{-1}=\gamma^{0}, \quad T \gamma^{k *} T^{-1}=-\gamma^{k}
$$

or

$$
T \gamma^{0} T^{-1}=-\gamma^{0}, \quad T \gamma^{k *} T^{-1}=\gamma^{k} .
$$

The relations (19) are the same as those obtained from the Dirac equation, whose already known solution is $T_{D}=e^{i \lambda} \gamma^{3} \gamma^{1}$, while from the relations (20) is obtained $T=T^{\prime}=w \gamma^{2} \gamma^{0}$, with $w \in \mathbb{C}^{*}=\mathbb{C} \backslash\{0\}$.

For $T=T^{\prime}$, applying $\tau$ twice, we arrive to:

$$
\hat{\psi}(t, \mathbf{x}) \rightarrow \hat{\psi}_{\tau}(t, \mathbf{x})=T^{\prime} \hat{\psi}(-t, \mathbf{x})^{*} \rightarrow T^{\prime}\left(T^{\prime} \hat{\psi}(t, \mathbf{x})^{*}\right)^{*}=T^{\prime} T^{\prime *} \hat{\psi}(t, \mathbf{x}) .
$$

and taking into account that

$$
T^{\prime} T^{*}=|w|^{2} \gamma^{2} \gamma^{0}\left(\gamma^{2} \gamma^{0}\right)^{*}=-|w|^{2} \gamma^{2} \gamma^{0} \gamma^{2} \gamma^{0}=|w|^{2} \gamma^{2} \gamma^{2} \gamma^{0} \gamma^{0}=-|w|^{2} 1,
$$

it follows that $\hat{\psi}_{\tau^{2}}=-\hat{\psi}$, by a similar argument to that used for $C$.

Thus, $T^{\prime} T^{*}=-1$, which implies $T^{\prime *}=-T^{\prime-1}$ and $w \in U(1)$. Then, $T^{\prime}=$ $e^{i \lambda} \gamma^{2} \gamma^{0}$ y $T^{\prime \dagger}=e^{-i \lambda} \gamma^{2} \gamma^{0}$.

\section{Matrix and operator CPT groups}

In summary, one has both the two sets of matrices:
a) $C_{D}= \pm \gamma^{2} \gamma^{0}$
$P_{D}= \pm i \gamma^{0}$
$T_{D}= \pm i \gamma^{3} \gamma^{1}$
b) $C_{D}= \pm i \gamma^{2} \gamma^{0}, \quad P_{D}= \pm i \gamma^{0}, \quad T_{D}= \pm \gamma^{3} \gamma^{1}$;

and the set: $C^{\prime}, P^{\prime}, T^{\prime}$, satisfying the subsidiary equation. But, only the matrices $C_{D}, P_{D}, T_{D}$, also satisfy the Dirac type equation. That is why they are the matrices which conform the matrix CPT group of the spin-3/2 field with mass.

If we take the zero mass limit of the Dirac type equation,

$$
i \gamma^{\alpha} \partial_{\alpha} \hat{\psi}(x)=0
$$


and analyze its behavior under parity, charge conjugation and time reversal, as we did for the subsidiary condition, we have, respectively:

$$
\begin{gathered}
i\left(P \gamma^{0} P^{-1} \partial_{0}-P \gamma^{i} P^{-1} \partial_{i}\right) \hat{\psi}_{\Pi}(x)=0, \\
\left(i \partial_{\mu}+q A_{\mu}\right)\left(C \gamma^{0}\right) \gamma^{\mu *}\left(C \gamma^{0}\right)^{-1} \hat{\psi}_{C}(x)=0 \\
i\left(\gamma^{0 *} \frac{\partial}{\partial t}-\gamma^{k *} \frac{\partial}{\partial x^{k}}\right) \hat{\psi}_{\tau}(x)=0 .
\end{gathered}
$$

From the above equations we can then obtain the corresponding restrictions on the $C, P$ and $T$ matrices, respectively:

$$
\begin{aligned}
P \gamma^{0} P^{-1}= \pm \gamma^{0}, \quad P \gamma^{k} P^{-1} & =\mp \gamma^{k} \\
C \gamma^{\mu T} C^{-1} & = \pm \gamma^{\mu} \\
T \gamma^{0} T^{-1}= \pm \gamma^{0}, \quad T \gamma^{k *} T^{-1} & =\mp \gamma^{k}
\end{aligned}
$$

These relations generate the same sets of matrices $C_{D}, P_{D}, T_{D}$ and $C^{\prime}, P^{\prime}, T^{\prime}$ which gave the subsidiary condition. But if we take into account that $\hat{\bar{\psi}} \hat{\psi}$ is the charge density operator, for $C=C^{\prime}$, it follows that:

$$
\begin{aligned}
(\hat{\bar{\psi}} \hat{\psi})_{C} & =\hat{\bar{\psi}}_{C} \hat{\psi}_{C}=-\bar{\eta}^{2} \hat{\psi}^{T} C^{\prime} C^{\prime} \hat{\bar{\psi}}^{T}=-\bar{\eta}^{2} C^{\prime 2}(\hat{\bar{\psi}} \hat{\psi})^{T} \\
& =-\bar{\eta}^{2} C^{\prime 2} \hat{\bar{\psi}} \hat{\psi}=(\bar{\eta} \eta)^{2} \hat{\bar{\psi}} \hat{\psi}=|\eta|^{4} \hat{\bar{\psi}} \hat{\psi}=-\hat{\bar{\psi}} \hat{\psi}
\end{aligned}
$$

from which $|\eta|^{4}=-1$, which is a contradiction. Hence, the matrix $C=C^{\prime}$ must be discarted.

It was demostrated in [1] that $C$ and $P$ must fulfill the relation:

$$
C\left(P^{-1}\right)^{T} C^{-1}=P
$$

while $C$ and $T$ are related by:

$$
C T^{*}=T C^{*}
$$

Due to the fact that each component of the vector spinor satisfies a Dirac type equation, the above relations also hold in our case. That is why the set of matrices: $C^{\prime}, P^{\prime}, T^{\prime}$ are also discarded for the massless case. 
In order to find the operator CPT group for the spin-3/2 field (with or without mass), we follow the same procedure developed in [1], for the case of the corresponding CPT group of the Dirac field.

Taking $\hat{A}$ and $\hat{B}$ as any of the operators $\hat{C}_{D}, \hat{P}_{D}$ and $\hat{T}_{D}$; and $\hat{\psi}$, as each component of the vector spinor $\hat{\psi}^{\mu}(x)$, the relations:

$$
\hat{A} \cdot \hat{\psi}=\hat{A}^{\dagger} \hat{\psi} \hat{A}
$$

and

$$
(\hat{A} * \hat{B}) \cdot \hat{\psi}=(\hat{A} \hat{B})^{\dagger} \hat{\psi}(\hat{A} \hat{B})
$$

can be defined.

Using the above expressions and with support in the matrix CPT group, through the formulas that link matrices with operators:

$$
\begin{array}{r}
P \hat{\psi}(t,-\mathbf{x})=\hat{P}^{\dagger} \hat{\psi}(t, \mathbf{x}) \hat{P}, \\
C \hat{\bar{\psi}}^{T}(x)=\hat{C}^{\dagger} \hat{\psi}(x) \hat{C}, \\
T \hat{\psi}(-t, \mathbf{x})^{*}=\hat{T}^{\dagger} \hat{\psi}(t, \mathbf{x})^{\dagger} \hat{T} ;
\end{array}
$$

the relations:

$$
\begin{array}{r}
\hat{P_{D}} * \hat{P_{D}}=-1, \quad \hat{C_{D}} * \hat{C_{D}}=1, \quad \hat{T_{D}} * \hat{T_{D}}=-1, \\
\hat{T_{D}} * \hat{P_{D}}=-\hat{P_{D}} * \hat{T_{D}}, \hat{C_{D}} * \hat{P_{D}}=\hat{P_{D}} * \hat{C_{D}}, \hat{C_{D}} * \hat{T_{D}}=\hat{T_{D}} * \hat{C_{D}},
\end{array}
$$

were obtained in [1]; from which it is possible to build, also using the property of associativity, the multiplication table for the operator CPT group.

It was also demostrated in [1] that only the second of the two solutions for the matrix group $\left(G_{\theta}^{(2)} \cong 16 E \cong D_{4} \rtimes \mathbb{Z}_{2}\right)$, is compatible with the operator group $\left(G_{\hat{\theta}} \cong Q \times \mathbb{Z}_{2}\right)$.

In summary, we showed that both the matrix and the operator CPT groups for the spin-3/2 field (with or without mass) coincide with the corresponding groups for the Dirac field.

\section{Acknowledgment}

This work was partially support by the project PAPIIT IN 118609-2, DGAPAUNAM, México. B. Carballo Pérez also acknowledge financial support from CONACyT, México. The authors thank Prof. O. S. Zandrón from IFIR, Rosario, Argentina, for suggesting this work. 


\section{References}

[1] M. Socolovsky, 2004, The CPT group of the Dirac Field, International Journal of Theoretical Physics, 43, 1941-1967; arXiv: math-ph/0404038.

[2] F. Riazuddin, 2000, A modern introduction to Particle Physics, Second Edition (World Scientific), 121-122.

[3] W. Rarita and J. Schwinger, 1941, On a Theory of Particles with HalfIntegral Spin, Physical Review, 60, 61. 